High Energy Phenomena in Relativistic Outflows III (HEPRO III)

International Journal of Modern Physics: Conference Series

Vol. 8 (2012) 209-219

(C) World Scientific Publishing Company

DOI: $10.1142 / \mathrm{S} 2010194512004618$

\title{
CLOSE BINARY PROGENITORS OF HYPERNOVAE
}

\author{
MAXIM V. BARKOV \\ Max-Planck-Institut für Kernphysik, \\ Saupfercheckweg 1, Heidelberg, D-69117, Germany \\ Space Research Institute RAS, \\ 84/32 Profsoyuznaya Street, Moscow, 117997, Russia \\ bmv@mpi-hd.mpg.de
}

\begin{abstract}
In this paper we propose a new plausible mechanism of supernova explosions specific to close binary systems. The starting point is the common envelope phase in the evolution of a binary consisting of a red super giant and a neutron star. As the neutron star spirals towards the center of its companion it spins up via disk accretion. Depending on the specific angular momentum of gas captured by the neutron star via the Bondi-Hoyle mechanism, it may reach millisecond periods either when it is still inside the common envelope or after it has merged with the companion core. The high accretion rate may result in strong differential rotation of the neutron star and generation of a magnetarstrength magnetic field. The magnetar wind can blow away the common envelope if its magnetic field is as strong as $10^{15} \mathrm{G}$, and can destroy the entire companion if it is as strong as $10^{16} \mathrm{G}$. The total explosion energy can be comparable to the rotational energy of a millisecond pulsar and reach $10^{52} \mathrm{erg}$. The result is an unusual type-II supernova with very high luminosity during the plateau phase, followed by a sharp drop in brightness and a steep light-curve tail. The remnant is either a solitary magnetar or a close binary involving a Wolf-Rayet star and a magnetar. When this Wolf-Rayet star explodes this will be a third supernovae explosion in the same binary. A particularly interesting version of the binary progenitor involves merger of a red super giant star with an ultra-compact companion, neutron star or black hole. In the case if a strong magnetic field is not generated on the surface of a neutron star then it will collapse to a black hole. After that we expect the formation of a very long-lived accretion disk around the black hole. The Blandford-Znajek driven jet from this black hole may drive not only hypernovae explosion but produce a bright X-ray transient event on a time scale of $10^{4} \mathrm{~s}$.
\end{abstract}

Keywords: gamma ray burst; hypernovae; X-ray flares.

\section{Introduction}

Millisecond pulsars are found in low mass binaries, and it is generally thought that they have been spun up via disk accretion [1, 2]. This origin implies mass increase by about $0.2 M_{\odot}$ compared to normal radio pulsars, whose masses are narrowly distributed around $1.35 M_{\odot}$ [3]. It is rather difficult to measure the mass of a millisecond pulsar, but the few available results agree with this prediction of the accretion model $[4,5,6]$. The most massive pulsar found to date, of almost $2 M_{\odot}$, is a millisecond pulsar [6]. The magnetic field of these millisecond pulsars is very low, down to $10^{9}$ Gauss. Most likely, their initial magnetic field was of similar strength 
to normal pulsars, but now it is buried under the layers of accreted matter $[7,1]$. The reason why these pulsars could not generate magnetar-strength magnetic field in the same way as in the core-collapse scenario is the very long time scale of spinning up compared to the viscous time-scale. As the result, the differential rotation remains weak and there is not enough energy for effective magnetic dynamo [8].

The rotational frequency of the fastest X-ray pulsar is $\sim 760 \mathrm{~Hz}[9]$, whereas the fastest known radio pulsar has the frequency of $641 \mathrm{~Hz}$ [10]. Most likely, it is the gravitational radiation losses what places the upper limit on the rotation rates because at high spin the r-mode oscillations become excited [11, 12]. H. Spruit [8] argued that this instability may also result in magnetic explosion. The idea is that the heating of NS, associated with these oscillations, reduces its viscosity and decouples its interior from the outer layers. Being most disturbed, the outer layers rapidly loose some of their angular momentum via gravitational radiation. This leads to strong differential rotation and generation of magnetar strength magnetic field in the NS interior. This field becomes unstable to buoyancy, emerges on the surface, and a magnetically driven pulsar wind rapidly extracts the rotational energy of the NS. In the work [8] proposed this as an alternative scenario for long GRBs. However, it is unlikely that a supernova-like event can accompany a GRB in this scenario. Although the wind energetics is sufficient, only a small fraction of this energy can be deposited into the companion star, simply because of its small geometrical crosssection. Moreover, recent results suggest that the amplitude of r-modes may saturate at a much lower level due to nonlinear interaction with other modes $[13,14,15]$.

A similar recycling of NS may occur during the common envelop (CE) phase, after the primary becomes a red super giant (RSG) [16, 17, 18, 19]. Due to the dynamic friction, the NS then spirals inside the RSG, accreting on its way. Now one can imagine two interesting outcomes of such process. First, the neutron star may accumulate too much mass and collapse into a black hole $(\mathrm{BH})$. This $\mathrm{BH}$ is likely to be rapidly rotating and drive a stellar explosion in the collapsar fashion $[20,21$, $22,23,24]$.

Second, the NS may first spin up to a millisecond period and drive a magnetic explosion of the type proposed by [8] but now inside the common envelope. The magnetar wind can keep energising such supernovae, producing a similar effect to radioactive decay $[25,26,27]$. High accretion rates may modify the NS recycle way. The accreted gas can form a massive rapidly rotating layer above the NS crust [28, 29]. The strong differential rotation between the layer and the NS core may result in the development of the Kelvin-Helmholtz instability when the NS crust melts down under the weight of the layer. This may lead to turbulence and strong amplification of the NS magnetic field.

\section{Inspiral dynamics and recycling of NS}

As the neutron spirals inside its giant companion it accretes mass and angular momentum. The accretion can proceed at the Eddington rate or at the much higher 
Bondi-Hoyle rate if the radiation generated during the collision of the accreted matter with NS or its accretion disk becomes trapped in the accretion flow. This depends on the position of the accretion shock, which depends of the efficiency of neutrino cooling [30, 31, 32]. Only if the accretion proceeds at the Bondi-Hoyle rate the recycling of NS is sufficiently fast and the explosion can occur.

The Bondi-Hoyle mass accretion rate is well approximated by the equation

$$
\dot{M}_{\mathrm{B}-\mathrm{H}}=\pi R_{\mathrm{A}}^{2} \rho_{\infty} v_{\infty},
$$

where $R_{A}$ is the accretion radius

$$
R_{\mathrm{A}} \approx 2 \beta \frac{a M}{M_{*}},
$$

where $\beta \simeq 0.8$. $R_{A}$ only weakly depends on the model of RSG.

We will assume that the specific angular momentum

$$
\left\langle j_{\mathrm{A}}\right\rangle=\frac{\eta}{4} \Omega R_{\mathrm{A}}^{2},
$$

where $\eta$ is a free parameter, which reflects our current ignorance.

Given the specific angular momentum we can estimate at what radius from NS the Bondi-Hoyle trapped gas will form an accretion disk,

$$
R_{\mathrm{c}}=\frac{\left\langle j_{\mathrm{A}}\right\rangle^{2}}{G M} \approx a \eta^{2} \beta^{4}\left(\frac{M}{M_{*}}\right)^{3} .
$$

For $R \gg R_{c}$ the accretion on NS may proceed in more or less spherical fasion as in the Bondi solution, where at $R_{\text {acc }}=0.25 R_{\mathrm{A}}$, for the polytropic index $\gamma=4 / 3$, the

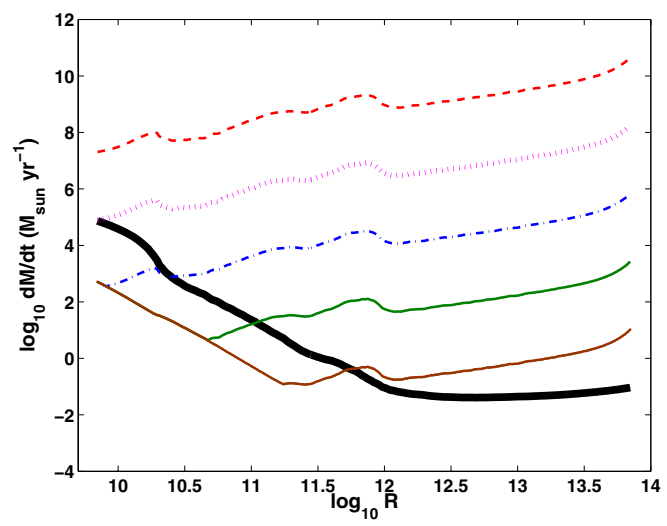

Fig. 1. Accretion rate as a function of the distance between the RSG and NS centers (we note that NS is accommodated by the RSG star) as obtained in the frameworks of the model (suggested by Heger at al. 2004) for $M_{\mathrm{rsg}}=25 M_{\odot}$. The critical accretion rates for the following values of the $\eta$ parameter are shown: $\eta=1,1 / 2,1 / 4,1 / 8$ and $1 / 16$ (from the up to the down). For these values, the interaction of the NS with the envelop prevents an efficient accretion to the NS, unless the Bondi-Hoyle rate, which is shown by the thick solid line, exceeds the critical value. For the higher accretion rates, the accretion occurs at the Bondi-Hoyle regime. 
accretion flow becomes supersonic. Its collision with the NS surface or the surface of its accretion disk creates a shock wave, which may not affect the mass accretion rate provided two conditions are satisfied.

Firstly, the radiation produced by the gas heated at the accretion shock should not be able to escape beyond $R_{\mathrm{A}}$. If it does the radiation pressure prevents the mass accretion rate to exceed the Edington rate. For this to occure $\dot{M}_{\mathrm{B}-\mathrm{H}}$ has to exceed the critical value

$$
\dot{M}_{\mathrm{cn} 1} \approx 1.1 \times 10^{-3}\left(\frac{R_{\mathrm{c}}}{10^{6} \mathrm{~cm}}\right)^{1.08} M_{\odot} \mathrm{yr}^{-1}
$$

Secondly, the shock has to be inside the sonic surface of the Bondi flow. Otherwise, the shock cannot be a part of the stationary solution and hence the Bondi-type accretion is not realised. This condition is satisfied when the $\dot{M}_{\mathrm{BH}}$ exceeds the critical value

$$
\dot{M}_{\mathrm{cn} 2} \approx 10^{4}\left(\frac{R_{\mathrm{acc}}}{10^{8} \mathrm{~cm}}\right)^{-2.7}\left(\frac{R_{\mathrm{c}}}{10^{6} \mathrm{~cm}}\right)^{4} M_{\odot} \mathrm{yr}^{-1} .
$$

As we shall see this condition is more restrictive. The critical rates are quite sensitive to the parameter $\eta$,

$$
\dot{M}_{\mathrm{cn} 1} \propto \eta^{2.16} \text { and } \quad \dot{M}_{\mathrm{cn} 2} \propto \eta^{8}
$$

Since $\dot{M}_{\mathrm{cn} 2}$ is very sensitive to $\eta$, Figure 1 shows $\dot{M}_{\mathrm{cn} 2}$ for few intermediate values of $\eta$ for the model of RSG with initial stellar mass $M_{\mathrm{ZAMS}}=25 M_{\odot}[33]$. One can see the fast accretion regime (at Bondi-Hoyle rates) is only possible when $\eta \lesssim 1 / 4$.

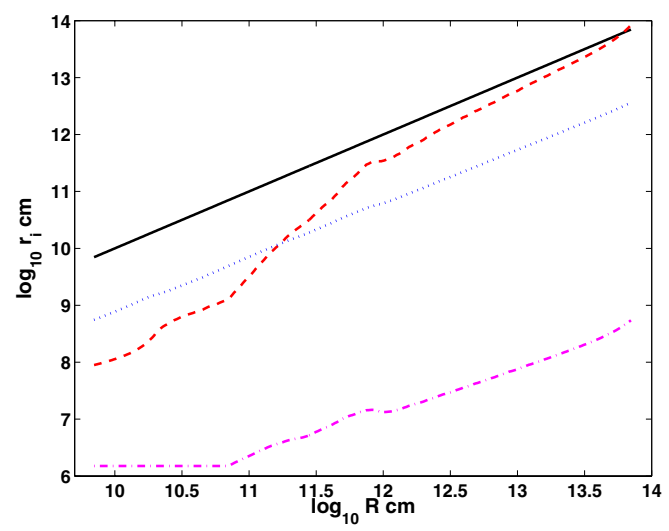

Fig. 2. The illustration of the typical linear scales of the scenario as a function of the distance between the RSG and NS centers. The calculations are performed in the frameworks of the same model as in Fig. 1 for a specific value of the $\eta$ parameter $\eta=0.1$. The orbital radius is shown by the solid line, the sonic point, $R_{\text {acc }}$, by the dotted line, the accretion shock radius by the dashed line, and the circularization radius, $R_{\mathrm{c}}$, by the dash-doted line. 
Once the accretion disk is formed the specific angular momentum of the gas settling on the NS surface is that of the last Keplerian orbit. In this case the star will reach the rotational rate $\Omega$ after accumulating

$$
\Delta M \simeq \frac{\Omega I}{j_{\mathrm{K}}} \simeq 0.27\left(\frac{M}{1.5 M_{\odot}}\right)\left(\frac{R}{10 \mathrm{~km}}\right)^{2}\left(\frac{P}{1 \mathrm{~ms}}\right) M_{\odot}
$$

\section{Magnetic field generation}

The main route the formation of "magnetars" is believed to be the core collapse of rapidly rotating stars. During the collapse, the proto-neutron star naturally develops strong differential rotation, with the angular velocity decreasing outwards. This allows generation of super-strong magnetic field via the $\alpha$ - $\Omega$-dynamo $[34,35]$ or magneto-rotational instability [36].

In our case, the conditions are rather different. The neutron star is already fullydeveloped, with a solid crust, when it enters the envelope of its companion. The typical NS crust has the thickness $h \approx 10^{5} \mathrm{~cm}$ and the density at the bottom $\rho \sim$ $10^{14} \mathrm{~g} \mathrm{~cm}^{-3}$ (see e.g. [37, 38]). The neutron star may have already been spinned up via accretion from the stellar wind, but its rotation rate may still be well below the critical value for the development of the gravitational wave instability. In any case, the accreted gas from the companion's envelope forms a very rapidly rotating layer above the crust. As long as the crust exists, this layer and the NS core are basically decoupled, and interact mainly gravitationally. A strong tangential discontinuity exists at the outer boundary of the crust [28, 29].

As the mass of the outer layer increases, the crust pressure goes up. Because of the degeneracy of the crust matter, its pressure depends mainly on its density, and only weakly on temperature. When the density reaches the critical value $\rho_{c r} \sim 10^{14} \mathrm{~g} \mathrm{~cm}^{-3}$, the crust begins to melt [39], and the barrier separating the NS core and its outer rotating layer disappears. If we ignore the centrifugal force that this generates, the NS accumulates mass comparable to the crust mass, $\sim 0.05 M_{\odot}$ before crust melting. The centrifugal force significantly reduces the effective gravitation acceleration and the above estimate for the layer mass is only a lower limit. Assuming $v_{\phi} \approx v_{K} \sim 10^{10} \mathrm{~cm} \mathrm{~s}^{-1}$ the rotational energy of the layer at the time of melting may well reach $\sim 10^{52}$ ergs. Once the crust has melted, the core and the layer can begin to interact hydromagnetically, with up to $E \sim 10^{52} \mathrm{ergs}$ of energy in differential rotation to be potentially released.

One possibility is viscous heating and neutrino emission. We can estimate the neutrino luminosity as $L_{\nu} \approx E / t_{d}$, where $t_{d}$ is the neutrino diffusion time. Following the analysis of $[38,40]$, this time can be estimated as

$$
t_{\mathrm{d}} \sim h \tau_{\nu} / c
$$

where $\tau_{\nu}=h \rho \Sigma_{\nu} / m_{p}$ is the optical depth of the hot envelope for the neutrino emission. Assuming equilibrium with the electron fraction $Y_{e}=0.05$, the mean neutrino cross-section is about $\Sigma_{\nu} \approx \sigma_{\nu} \epsilon_{\nu} k T_{e} / m_{e}^{2} c^{4}$, where $\sigma_{\nu}=1.7 \times 10^{-44} \mathrm{~cm}^{2}$ 
[41] and $\epsilon_{\nu} \approx 3.15 k T_{e}$ is the mean energy of the neutrinos [42]. The temperature can be estimated as

$$
T_{e} \approx\left(E / 4 \pi a_{r} h R_{\mathrm{NS}}^{2}\right)^{1 / 4} \approx 10^{12} E_{52}^{1 / 4} h_{5}^{-1 / 4} R_{\mathrm{NS}, 6}^{-1 / 2} \mathrm{~K},
$$

where $a_{r}=7.564 \times 10^{-15} \mathrm{erg} \mathrm{cm}^{-3} \mathrm{~K}^{-4}$ is the radiation constant. The corresponding diffusion time is quite short,

$$
t_{\mathrm{d}} \sim 0.1 h_{5}^{3 / 2} \rho_{14} E_{52}^{1 / 2} / R_{\mathrm{NS}, 6} \mathrm{~s} .
$$

We can estimate the maximal neutrino luminosity as $L_{\nu, \max } \approx E / t_{\mathrm{d}} \sim 10^{53} \mathrm{erg} \mathrm{s}^{-1}$.

Another process is development of the Kelvin-Helmholtz instability, production of turbulence and amplification of the magnetic field. For the Kelvin-Helmholtz instability to develop the Richardson number $J$ has to be below than $1 / 4$ [43]. In our case

$$
J \approx \frac{g h}{v_{\phi}^{2}} \approx 0.2 R_{\mathrm{NS}, 6} h_{5}
$$

where $g \approx G M_{N S} / R_{N S}^{2}$ is the gravitational acceleration and we use $M_{N S}=1.5 M_{\odot}$. Thus, the instability condition is marginally satisfied. Once the turbulence has developed the magnetic field amplification is expected to proceed as discussed in [34]. The e-folding time is given by the eddy turn-over time, which one would expect to be below the rotation period of the outer layer. Thus, strong magnetic field can be generated on a time shorter than the viscous and the neutrino diffusion time scales $^{\mathrm{a}}$ This conclusion is supported by the recent numerical simulations of similar problems, which show that a time as short as $0.03 \mathrm{~s}$ can be sufficient to generate a dynamically strong magnetic field $[45,46]$. The strong toroidal magnetic field can suppress the instability if $\rho v^{2}<B^{2} / 4 \pi[43]$ or if $B>3 \times 10^{17} \mathrm{G}$. This is likely to determine the saturation strength of the magnetic field. Such a strong magnetic field is also buoyant [8] and will emerge from under the NS surface into the surrounding accretion flow.

\section{BH formation on $\mathrm{CE}$ phase}

If on the stage of CE the strong magnetic field in the surface of NS is not generated NS can collapse to the BH. This could result in collapsar [21] fashion hypernovae explosion $[22,24]$. The main feature of such an explosion is that it happens in the extended and rarefied envelop of RSG and no GRB flare can be formed [27]. Such a merger can be divided into tree phases. During the first phase, the compact object spirals inside the extended envelope of the normal star and spins it up via deposition of its orbital angular momentum. The compact star also increases its mass and spin via the Bondi-type accretion. According to the simulations of [22], during the last 500 s of the inspiral the compact star can accumulate up to $3.5 M_{\odot}$ forming the $\mathrm{BH}$.

a The viscous dissipation timescale is very long $t_{v i s} \approx 10^{8} R_{N S, 6}^{23 / 4} T_{9}^{2} \mathrm{~s}[44]$. 
Thus, the mean accretion rate is less than $10^{-2} M_{\odot} / \mathrm{s}$ implying inefficient neutrino heating.

The second stage begins when the compact star approaches the center of its RSG companion and the accretion rate increases. In the work [22] it was found that in the case of $16 M_{\odot}$ companion the neutrino annihilation mechanism can operate for around 60 seconds and release about $10^{52} \mathrm{erg}$. This is more than enough to drive a supernova explosion due to collapsar scenario. For the companion mass below $8 M_{\odot}$ the neutrino heating is too weak and the second phase is absent.

The third phase occurs if the possible explosion during the second phase is highly non-spherical and does not remove the equatorial layers of the companion. During this phase the compact object, already a black hole, accretes these layers, which have been spin up during the first phase. Assuming that the mass of the compact star is small compared to the mass of its RSG companion, its orbital angular momentum can be found via the Keplerian law

$$
J_{c}(R)=M_{c} \sqrt{G M_{*}(R) R}
$$

where $M(R)$ is the WR mass inside the radius $R$. As the compact star moves from the radius $R+d R$ to $R$, it transfers the angular momentum $d J_{c}(R)$ to the mass $d M$ of the envelope located between $R$ and $R+d R$. Thus, the specific angular momentum of the envelope after the merger can be estimated as

$$
l \simeq \frac{d J_{c}}{d M_{*}}=\frac{d J_{c} / d R}{d M_{*} / d R} .
$$

For the Bethe's model [47], we have

$$
l \simeq \frac{M_{c}}{2}\left(\frac{G R}{M_{*}(R)}\right)^{1 / 2}\left(1+\ln \left(R / R_{c}\right)\right) .
$$

This angular momentum is smaller than the local Keplerian value if $M\left(R_{*}\right)>$ $M_{c}\left(1+\ln R / R_{c}\right) / 2$. Thus, provided that $M_{*}\left(R_{*}\right) \gg M_{c}$, only the very external layers of the common envelop will be lost as the result of the merger. For $R=R_{*}$ this equation gives

$$
l \simeq 5.2 \times 10^{18} \frac{M_{c}}{2 M_{\odot}}{\frac{R_{s}}{R_{\odot}}}^{1 / 2}{\frac{M_{*}}{10 M_{\odot}}}^{-1 / 2} \mathrm{~cm}^{2} \mathrm{~s}^{-1} .
$$

In the $\alpha$-model, the accretion time scale of the disk with such angular momentum can be estimated via

$$
\begin{gathered}
t_{d} \simeq \frac{1}{\alpha \delta^{2}} \frac{l^{3}}{\left(G M_{*}\right)^{2}}= \\
\simeq 8000\left(\frac{\alpha \delta^{2}}{0.01}\right)^{-1}\left(\frac{R_{*}}{R_{\odot}}\right)^{3 / 2}\left(\frac{M_{c}}{2 M_{\odot}}\right)^{2}\left(\frac{M_{*}}{10 M_{\odot}}\right)^{-7 / 2} \mathrm{~s},
\end{gathered}
$$

where $\delta \sim H_{d} / R_{d}$ is relative thickness of the accretion disk. 
To find the mass accretion rate as a function of time we note that

$$
\dot{M}=\frac{d M_{*}}{d t_{d}}=\frac{d M_{*} / d R}{d t_{d} / d R} .
$$

Using Eqs. $(12,10)$ to evaluate $d M_{*} / d R$ and $d t_{d} / d R$ we obtain

$$
\dot{M} \simeq \frac{2}{3} \frac{M_{*}}{\ln \left(R_{s} / R_{c}\right)} \frac{1}{t} \simeq 1.45 \frac{M_{*}}{10 M_{\odot}} \frac{t^{-1}}{1 \mathrm{~s}} \frac{M_{\odot}}{\mathrm{s}} .
$$

Thus, with a time scale of $10^{3}-10^{4}$ s the mass accretion rate is very low, $\dot{M} \simeq 10^{-3} \div$ $10^{-4} M_{\odot} \mathrm{s}^{-1}$, ruling out the neutrino mechanism and leaving the BZ mechanism as a clear favourite to form jets. Indeed, the maximum possible amount of magnetic flux that can be accumulated by the black hole is given by the balance of magnetic pressure and the gas pressure of the accretion disk,

$$
\frac{B_{\max }^{2}}{8 \pi} \simeq P_{g} \simeq \rho c_{a}^{2}
$$

where $c_{a}$ is the sound speed. If we utilize the model of the $\alpha$-disk and estimate the magnetic field strength at the gravitational radius, then the corresponding magnetic flux will be

$$
\Psi_{\text {max }} \simeq 3 \times 10^{29} \frac{\alpha \delta}{0.03}^{-1 / 2} \frac{M_{*}}{10 M_{\odot}} \dot{M}_{1}^{1 / 2} \mathrm{G} \mathrm{cm}^{2},
$$

where $\dot{M}_{1}$ is the mass accretion rate in the units of $M_{\odot} /$ s. Even for $\dot{M}_{1}$ as small as $10^{-4}$ this equation gives the substantial value of $\Psi_{\max } \simeq 3 \times 10^{27} \mathrm{G} \mathrm{cm}^{2}$ and according to [48] the BZ power, $\dot{E}_{B Z} \simeq 2.2 \times 10^{49} \mathrm{erg} / \mathrm{s}$, is more than sufficient to explain the X-ray observations, allowing the magnetic field to be even weaker than suggested by Eq.14.

The BZ active phase can be long enough to allow the jet to leave from the envelop of disrupted RSG. In fact, such a long time scale suggests the possibility of the formation of X-ray transients on time scales $\sim 10^{4} \mathrm{~s}$ accompanying the hypernovae explosion. This model is complimentary to the model of extended emission of short Gamma-ray Bursts [49].

\section{Discussion}

In a close binary system formed by a normal star and NS, the normal star in the stage of Roche lobe overflow, produces a strong wind through the $1^{\text {th }}$ Lagrangian point. When the NS approaches too close, the CE stage has to begin. At this epoch we can expect a very dense stellar wind with a mass loss rate at the level of $\sim 1 M_{\odot} \mathrm{yr}^{-1}$. During the CE stage the fast accretion to NS leads to a recycling of the NS up to ms periods. This rotation can overtake the stability limit and GW radiation can start to be very efficient. Thus, the outer layers of NS slowdown its rotation and a strong magnetic field could be generated in the vicinity of NS [8]. In this way, an ideal configuration that could explain of anomalously bright $\mathrm{SN}$ is formed. In the 
frameworks of our scenario [27], we can explain the big amount of hydrogen in the external envelop and anomalously intense stellar wind.

One of the important details in this model is that the explosion occurs strongly non-central, when NS is recycled till the limiting frequencies before reaching the center of RSG star. The pressure in the center of RSG is of the order of $10^{19} \mathrm{erg}$ $\mathrm{cm}^{-3}$ and can reach $10^{27}$ erg $\mathrm{cm}^{-3}$ at pre-supernovae stage, thus the pressure in the bubble blown by jets could be estimated as

$$
P_{b u b} \approx \frac{3 L t}{4 \pi R^{3}} \approx 8 \times 10^{17} L_{48} v_{s h,-1}^{-1} R_{10}^{-2}{\mathrm{dyn} \mathrm{cm}^{-2}}
$$

where $L$ is jet luminosity and $t$ is time from the explosion moment, for the following epoch $t \approx R / v_{s h}$, here $R$ is distance between NS and RSG centers, $v_{s h}$ the shock wave speed in speed of light units $c$. We can see that the pressure in the bubble is smaller than the pressure in the center of RSG if the explosion occurs at the distance of $R_{10}>1$, when the shock wave cannot destroy the stellar $\mathrm{C} / \mathrm{O}$ core. If the explosion occurs in the stellar center vicinity, the whole RSG can be destroyed by the explosion.

In the case of the remote explosion, although the mass of the RSG envelop can be bigger than the mass of the $\mathrm{C} / \mathrm{O}$ core and $M_{N S}$, the binary system cannot be destroyed, since the outer envelop does not influence gravitationally on the bounded system of $\mathrm{NS}+\mathrm{C} / \mathrm{O}$ core.
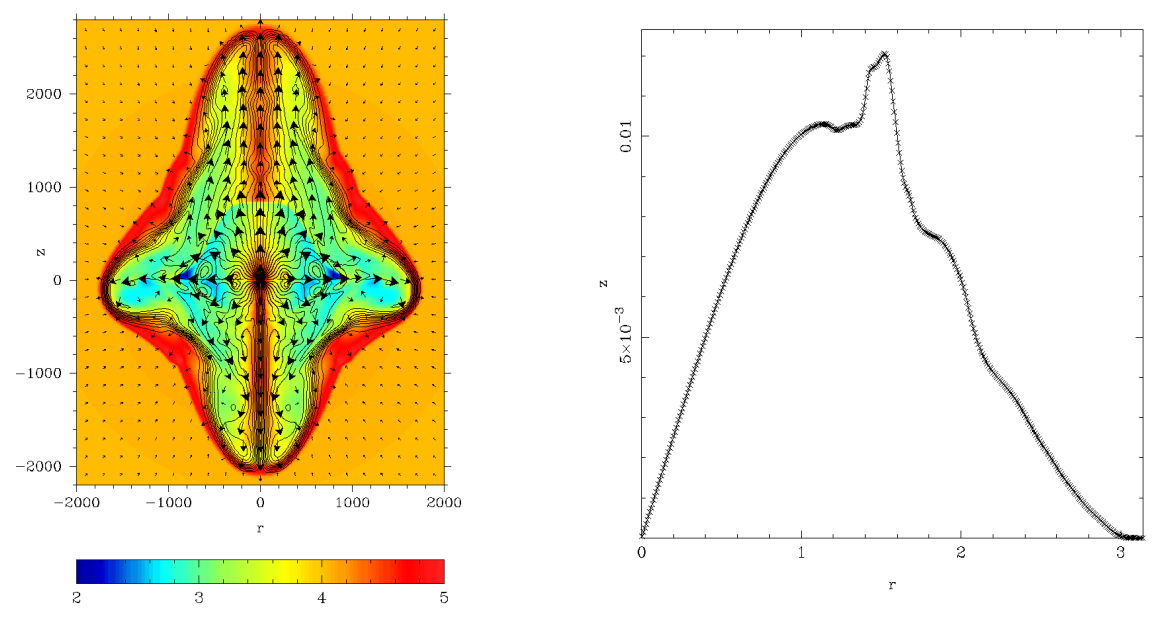

Fig. 3. The distribution of the density (left panel) and the zenith angle dependence of the energy flux at the distance of $110 \mathrm{~km}$ (right panel) are shown for the moment $0.056 \mathrm{~s}$ after the magnetically driven explosion. The initial density was uniform, and the magnetic field was assumed to be a shifted dipole field $B=3 \times 10^{15} \mathrm{G}$ (see the contour lines in the left panel). The velocity map is shown by arrows in the left panel. It may be seen that the expected released energy flux is strongly anisotropic. 
We note that the magnetic field topology is very important. Since the dynamo mechanism can generate multiples configurations, a superposition of dipole and quadrupole magnetic field is expected to produce a shifted dipole field configuration. The shifted dipole model has important features since it should automatically lead to an unsymmetrical explosion and anisotropy energy realize (another examples of one side jets one can find in the works $[24,50])$. Detailed numerical simulations display two side jet configurations but with significantly different power in jets (the energy ratio is close to 2:1 -see Fig.3-). This allows to estimate the kick for NS as

$$
v_{\text {kick }} \leq \frac{E_{t o t}}{3 M_{N S} c} \approx 370 E_{t o t, 52} \mathrm{~km} \mathrm{~s}^{-1}
$$

here $E_{t o t, 52}=E_{t o t} / 10^{52}$ ergs.

A particularly interesting version of the binary progenitor involves merger of a RSG star with an ultra-compact companion, neutron star or black hole. In the case a strong magnetic field is not generated then we expect the formation of very longlived accretion disks around the black hole. The Blandford-Znajek driven jet from this black hole may drive not only a hypernovae explosion like anomalously bright SN2006gy [51], which is an alternative to the pulsation pair instability supernovae [52], but also produce a bright X-ray transient event on the time scale $10^{4} \mathrm{~s}$.

\section{Acknowledgments}

Author is thankful to S.S.Komissarov V.Bosch-Ramon, N.Ikhsanov, and Y.Levin for fruitful discussions, as well as to A.Heger for providing models of RSGs. The calculations were fulfilled at cluster of Moscow State University "Chebyshev". This research was funded by STFC under the Rolling Programme of Astrophysical Research at Leeds University.

\section{References}

1. M. A. Alpar et al., Nature 300, 728 (1982).

2. A. M. Archibald et al., Science 324, 1411 (2009).

3. S. E. Thorsett and D. Chakrabarty, Astrophys. J. Lett. 512, 288 (1999).

4. V. M. Kaspi, J. H. Taylor and M. F. Ryba, Astrophys. J. Lett. 428, 713 (1994).

5. B. A. Jacoby et al., Astrophys. J. 629, L113 (2005).

6. P. B. Demorest et al., Nature 467, 1081 (2010).

7. G. S. Bisnovatyi-Kogan and B. V. Komberg, Soviet Astronomy 18, 217 (1974).

8. H. C. Spruit, Astron. Astrophys. 341, L1 (1999).

9. D. Chakrabarty et al., Nature 424, 42 (2003).

10. D. C. Backer et al., Nature 300, 615 (1982).

11. S. L. Shapiro and S. A. Teukolsky, in Black holes, white dwarfs, and neutron stars: The physics of compact objects (1983).

12. Y. Levin, Astrophys. J. Lett. 517, 328 (1999).

13. P. Arras et al., Astrophys. J. Lett. 5911129 (2003).

14. J. Brink, S. A. Teukolsky and I. Wasserman, Phys. Rev. D 71, 6, 064029 (2005).

15. R. Bondarescu, S. A. Teukolsky and I. Wasserman, Phys. Rev. D 76, 6, 064019 (2007). 
16. G. S. Bisnovatyi-Kogan and R. A. Syunyaev, Astronomicheskii Zhurnal 48, 881 (1971).

17. B. Paczynski, Common Envelope Binaries, in Structure and Evolution of Close Binary Systems, eds. P. Eggleton, S. Mitton and J. Whelan (IAU Symposium, 1976), p. 75

18. A. V. Tutukov and L. R. Yungelson, Acta Astronomica 29, 665 (1979).

19. K. A. Postnov and L. R. Yungelson, Living Reviews in Relativity 9, 6(2006).

20. C. L. Fryer and S. E. Woosley, Astrophys. J. Lett. 501, 780 (1998).

21. A. I. MacFadyen and S. E. Woosley, Astrophys. J. Lett. 524, 262 (1999).

22. W. Zhang and C. L. Fryer, Astrophys. J. Lett. 550, 357 (2001).

23. S. Nagataki, Astrophys. J. Lett. 704, 937 (2009).

24. M. V. Barkov and S. S. Komissarov, Mon. Not. R. Astron. Soc. 401, 1644 (2010).

25. S. E. Woosley, Astrophys. J. 719, L204 (2010).

26. D. Kasen and L. Bildsten, Astrophys. J. Lett. 717, 245 (2010).

27. M. V. Barkov and S. S. Komissarov, Mon. Not. R. Astron. Soc. 415, 944 (2011).

28. N. A. Inogamov and R. A. Sunyaev, Astronomy Letters 25, 269 (1999).

29. N. A. Inogamov and R. A. Sunyaev, Astronomy Letters 36, 848 (2010).

30. J. C. Houck and R. A. Chevalier, Astrophys. J. Lett. 376, 234 (1991).

31. R. A. Chevalier, Astrophys. J. 411, L33 (1993).

32. R. A. Chevalier, Astrophys. J. Lett. 459, 322 (1996).

33. A. Heger et al., Presupernova Evolution of Rotating Massive Stars and the Rotation Rate of Pulsars, in Stellar Rotation, eds. A. Maeder and P. Eenens (IAU Symposium, 2004), p 591.

34. R. C. Duncan and C. Thompson, Astrophys. J. 392, L9 (1992).

35. C. Thompson and R. C. Duncan, Astrophys. J. Lett. 408, 194 (1993).

36. A. Burrows et al., Astrophys. J. Lett. 664, 416 (2007).

37. O. Y. Gnedin, D. G. Yakovlev and A. Y. Potekhin, Mon. Not. R. Astron. Soc. 324, 725 (2001).

38. J. M. Lattimer and M. Prakash, Phys. Report 442, 109 (2007).

39. E. F. Brown, The Astrophysical Journal 531, 2, 988 (2000).

40. M. V. Barkov, in volume 1054 of American Institute of Physics Conference Series, ed. M. Axelsson (2008), p. 79.

41. J. N. Bahcall, Physical Review 136, 1164 (1964).

42. T. A. Thompson, A. Burrows and B. S. Meyer, Astrophys. J. Lett. 562, 887 (2001).

43. S. Chandrasekhar, Hydrodynamic and hydromagnetic stability (Oxford, 1961).

44. S. L. Shapiro, Astrophys. J. Lett. 544, 397 (2000).

45. M. Obergaulinger et al., Astron. Astrophys. 498, 241 (2009).

46. L. Rezzolla et al., arXiv:1101.4298 (2011).

47. H. A. Bethe, Reviews of Modern Physics 62, 801 (1990).

48. M. V. Barkov and S. S. Komissarov, Mon. Not. R. Astron. Soc. 385, L28 (2008).

49. M. V. Barkov and A. S. Pozanenko, Mon. Not. R. Astron. Soc., pages 1381 (2011).

50. R. V. E. Lovelace et al., arXiv e-prints (2010).

51. N. Smith et al., Astrophys. J. Lett. 666, 1116 (2007).

52. S. E. Woosley, S. Blinnikov and A. Heger, Nature 450, 390 (2007). 